\title{
UN MÉTODO RÁPIDO Y SIMPLE PARA EVALUAR LA CALIDAD BIOLÓGICA DE LAS AGUAS CORRIENTES BASADO EN EL DE HELLAWELL (1978)
}

\author{
J. Alba-Tercedor y A. Sánchez-Ortega \\ Departamento de Biología Animal, Ewlogía y Genética. Facultad de Ciencias, Universidad de Granada. España.
}

Palabras clave: rivers, maroinvertebrate, biotic index, Iberian Peninsula.

\begin{abstract}
A SIMPLE AND QUICK METHOD TO EVALUATE BIOLOGICAL QUALITY OF RUNNING FRESHWATER BASED ON HELLAWELL (1978)

A new approach to the the original B.M.W.P. (Hellawell, 1978) have been performed. Most of the macroinvertebrate families living in the Iberian Peninsula have been added to the original index and some of the scores have been changed. By comparison with some others biotic and diversity indexes, the different values of the new approach (B.M.W.P') have been correlated with quality classes and rate of pollution.
\end{abstract}

\section{INTRODUCCIÓN}

El creciente incremento de las alteraciones de los cursos de agua y la sensibilidad a este problema por parte de los organismos competentes, ha hecho que en todos los paises desarrollados se pongan en marcha programas de control y vigilancia de la calidad de las aguas. Para ello se han desarrollado numerosos métodos o índices que tratan de interpretar la situación real, o grado de alteración de los ecosistemas acuáticos. Unos se basan exclusivamente en análisis de las condiciones químicas, que si bien «en principio» son de una gran precisión, presentan el problema de ser testigos, tan sólo, de las condiciones instantáneas de las aguas. Por el contrario los llamados índices biológicos informan de la situación tanto momentánea como de lo acontecido algun tiempo antes de la toma de muestras.

En general los medios más estables están más diversificados (si bien pequeñas alteraciones pueden no afectar a la diversidad e incluso inducen incrementos de la misma, WARD \& STANDFORD, 1983), ya que las especies intolerantes desaparecen o disminuyen sus efectivos, mientras que las

Limnética, 4: 51-56 (1988)

(C) Asociación Española de Limnología, Madrid, Spain tolerantes $\mathrm{u}$ «oportunistas» los incrementan. Es por ello que las medidas de diversidad se han utilizado para detectar la contaminación.

El problema estriba en hacer corresponder a un determinado valor de índice un significado respecto de la calidad. Autores como WhiLhm \& DORRIS (1968), tras estudiar las variaciones del índice de Shannon (Shannon \& WeAVER, 1949) en aguas con distintos grados de contaminación, consideraron intervalos con una significación respecto de la calidad de las aguas. Así, según estos autores, valores superiores a 3 indican aguas limpias, entre 1 y 3 aguas ligeramente contaminadas y los inferiores a 1 corresponden a aguas intensamente contaminadas. $\mathrm{La}$ aplicación de este criterio es problemática, ya que por un lado diferencia pocos grados de contaminación, y por otro, los valores de la diversidad varían según el nivel taxonómico considerado (Washington, 1984; Prat et al., 1984).

En los últimos años se viene desarrollando en nuestro país un creciente interés por el estudio y aplicación de índices biológicos (DomeZAIN $\boldsymbol{e t}$ al., en prensa; González del TÁNAGo et al., 1979; García de Jalón \& González del Tánago, 1986; López Llaneza, 1984; Prat et al., 1984, 
1985). Los índices biológicos que se vienen utilizando requieren identificaciones de los organismos, en parte a nivel específico (CHANDLER, 1970; García de JALÓN \& GonZÁlez del TánAGO, 1986; PrAT et al., 1984, 1985) y/o genérico (T.V.: Tuffery \& Verneaux, 1967; E.B.I.: Woodiwiss, 1978), lo cual dificulta su aplicación en nuestro país, dada la problemática de identificación de algunos grupos de macroinvertebrados acuáticos, y sobre todo por la falta de claves de identificación adaptadas a la fauna ibérica.

\section{ÍNDICE B.M.W.P'}

Por las razones expuestas, se ha emprendido la tarea de buscar un índice que siendo «fiable», fuese a su vez de fácil aplicación. En una primera aproximación se observó que los valores del índice B.M.W.P. (Hellawell, 1978) oscilaban de forma similar a como lo hacían los valores de diversidad (H), T.V. y E.B.I. La fácil aplicación de éste índice, al no requerir identificaciones más que a nivel de familia, lo hizo

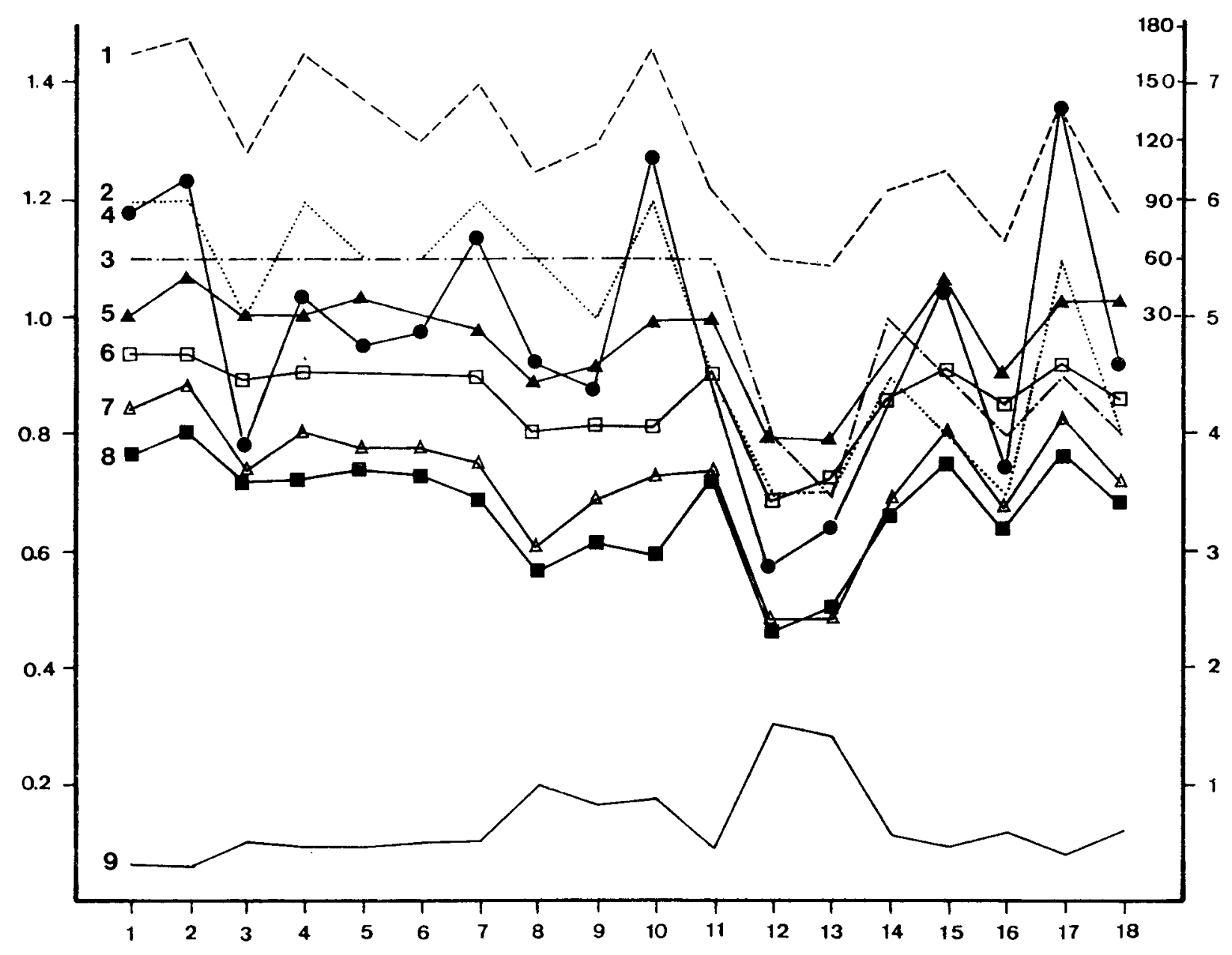

Figura 1.- Comparación entre los valores obtenidos para el B.M.W.P'. y diferentes índices de diversidad y de calidad de las aguas. (Los datos corresponden a estaciones de muestreo situadas a lo largo de la Cuenca del Río Adra - ALBA-TERCEDOR et al., en prensa -). 1: B.M.W.P'.; 2: E.B.I.; 3: T.V.; 4: Margalef; 5: TU; 6: PIE; 7: H; 9:D.

Comparison between differents B.M.W.P'. values with values of diversity and water quality indexes. (Data correspond to sarnpling stations situated along the Adra river bassin - ALBA-TerCEDOR et al., in press -)1: B.M.W.P'; 2: E.B.I.; 3: T.V.; 4: Margalef; 5: TU; 6: PIE; 7: H; 9: D. 
recomendable para el seguimiento de alteraciones en los ríos (Domezain et al., en prensa). Sin embargo en la tabla original, no estaban incluidas muchas de las familias de macroinvertebrados que son comunes en la Península Ibérica, las cuales fueron incluidas asignándoles una puntuación (Alba-TercedoK \& Jiménez Millán, 1985, 1987).

De este modo se disponía ahora de un método de evaluación de alteraciones de los cursos de agua, identificando los macroinvertebrados tan sólo a nivel de familia. Sin embargo, se necesitaba asignar a los valores obtenidos al aplicar éste método una determinada significación de la calidad de las aguas. Es por ello que posteriormente (Alba-Tercedor et $a l$., en prensa) se hizo un estudio comparativo entre los valores obtenidos, con la nueva acotación del índice de Hellawell (que hemos llamado B.M.W.P'), con diferentes índices de diversidad recomendados por WASHINGTON (1984) [Hulbert, 1971 (PIE); KeEFE, 1976 (TU); Mcintosh, 1967 (M); Shannon \& Weaver, 1949 (H); Simpson, 1949 (D)], el de Margalef (1951) y los índices biólogicos T.V y E.B.I. (fig. 1); así como el grado de correlación entre ellos. Para ello se obtuvo una matriz de correlación lineal y mediante la misma se realizó un análisis «cluster» por agrupación simple (con ayuda del programa BMDP-1M, DixON, 1983). Este análisis separó dos grupos (fig. 2); por un lado el formado por los índices de diversidad y por otro los índices biológicos, quedando unido a estos últimos el índice de Margalef.

El índice B.M.W.P. (Hellawell, 1978) ajustado y modificado por ALBA-TERCEDOR \& JIMÉNEZ (1985; 1987) (B.M.W.P'), si bien habia demostrado ser muy sensible a las variaciones de calidad de las aguas, sus valores no se habian identificado con grados de alteración.

Siguiendo las normas y recomendaciones del III Seminario técnico organizado por la Comunidad Europea sobre «Métodos biológicos de evaluación de la calidad del agua» (GHETTI \& BonAzZI, 1980) y al estar los valores del B.M.W.P' íntimamente correlacionados con los del E.B.I. (figs. 1 y 2), los acotamos en cinco clases de calidad (basadas en las descritas por Ghetтi et al. 1983), que se esquematizan en la tabla 2.

El valor del índice se obtiene por la suma de puntuación correspondiente a cada familia que habita en el tramo objeto de estudio, según se expone en la tabla 1.

En la primera aproximación a este índice (Alba-Tercedor \& Jiménez Millán, 1985; 1987) se incrementó el número de taxones que contemplaba la tabla original de Hellawell (1978), manteniendo las puntuaciones originales. Sin embargo en ésta que presentamos ahora se puntúa menos la presencia de taxones como Ephemerellidae, Caenidae, las familias de heterópteros y algunas familias de coleópteros.

El problema de establecer límites estrictos de calidad de aguas implica la necesidad de reconocer situaciones intermedias entre unos y otros. Es por ello que aquellos valores del B.M.W.P' que queden cinco unidades por exceso o por

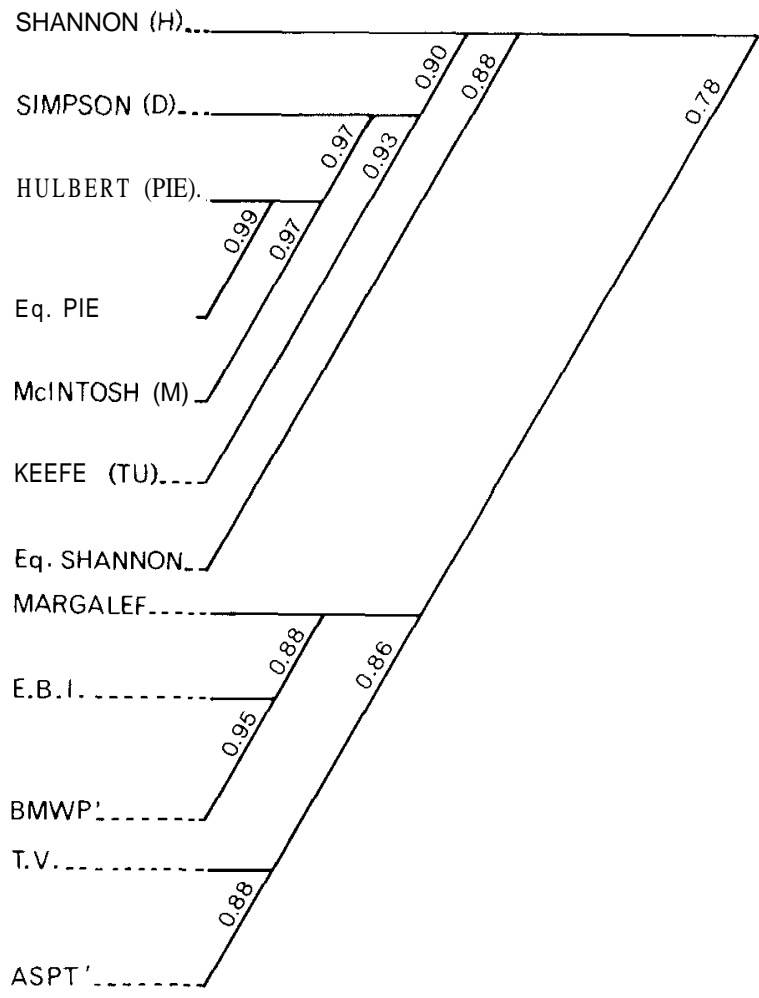

Figura 2.- Dendrograma de afinidades entre diferentes índices de diversidad y de calidad de aguas. Los números indican el coeficiente de correlación lineal.

Affinities tree between differents diversity and water quality indexes. Numbers correspond to linear regression coefficients. 
Tabla 1.- Puntuaciones asignadas a las diferentes familias de macroinvertebrados acuáticos para la obtención del B.M.W.P'. The B.M.W.P'. score system.

Siphlonuridae, Heptageniidae, Leptophebiidae, Potamanthidae, Ephemeridae

Taeniopterygidae, Leuctridae, Capniidae, Perlodidae, Perlidae

Chloroperlidae

Aphelocheiridae

Phryganeidae, Molannidae, Beraeidae, Odontoceridae, Leptoceridae, Goeridae

Lepidostomatidae, Brachycentridae, Sericostomatidae

Athericidae, Blephariceridae

\section{Astacidae}

Lestidae, Calopterygidae, Gomphidae, Cordulegasteridae, Aeshnidae

Corduliidae, Libellulidae

Psychomyiidae, Philopotamidae, Glossosomatidae

Ephemerellidae

Nemouridae

Rhyacophilidae, Polycentropodidae, Limnephilidae

Neritidae, Viviparidae, Ancylidae

Hydroptilidae

Unionidae

Corophiidae, Gammaridae

Platycnemididae, Coenagriidae

Oligoneuriidae

Dryopidae, Elmidae, Helophoridae, Hydrochidae, Hydraenidae, Clambidae

Hydropsychidae

Tipulidae, Simuliidae

Planariidae, Dendrocoelidae, Dugesiidae

Baeiidae, Caenidae

Haliplidae, Curculionidae, Chrysomelidae

Tabanidae, Stratiomyidae, Empididae, Dolichopodidae, Dixidae

Ceratopogonidae, Anthomyidae, Limoniidae, Psychodidae

Sialidae

Piscicolidae

Hidracarina

Mesoveliidae, Hydrometridae, Gerridae, Nepidae, Naucoridae, Pleidae

Notonectidae, Corixidae

Helodidae, Hydrophilidae, Hygrobiidae, Dytiscidae, Gyrinidae

Valvatidae, Hydrobiidae, Lymnaeidae, Physidae, Planorbidae

Bithyniidae, Bythinellidae, Sphaeridae

Glossiphoniidae, Hirudidae, Erpobdellidae

Asellidae, Ostracoda

Chironomidae, Culicidae, Muscidae, Thaumaleidae, Ephydridae 
Tabla 2.- Clases de calidad, significación de los valores del B.M.W.P'. y colores a utilizar en representaciones cartográficas. Quality class, meaning of the B.M.W.P'. values and colours to use in cartographic representations.

\begin{tabular}{|c|c|c|c|}
\hline Clase & Valor $\left(B M W P^{\prime}\right)$ & Significado & Color \\
\hline & $>150$ & Aguas muy limpias & \\
\hline I & $101-120$ & Aguas no contaminadas o no alteradas de modo sensible & Azul \\
\hline II & $61-100$ & Son evidentes algunos efectos de contaminación & Verde \\
\hline III & $36-60$ & Aguas contaminadas & Amarillo \\
\hline IV & $16-35$ & Aguas muy contaminadas & Naranja \\
\hline $\mathrm{V}$ & $<15$ & Aguas fuertemente contaminadas & Rojo \\
\hline
\end{tabular}

defecto de los límites establecidos en la tabla 2 , se deben considerar intermedios entre dos clases de calidad. Por tanto a la hora de realizar mapas de calidad de aguas, estos casos han de presentarse en trazos discontínuos, alternando los colores representativos de las clases correspondientes de calidad.

La fiabilidad de los resultados obtenidos con el B.M.W.P', la rapidez de su obtención y la facilidad de su utilización (al no requerir más que unos conocimientos taxonómicos muy elementales) convierten a éste índice en una magnífica «herramienta» para el seguimiento y gestión de cuencas por personal técnico, con un importante ahorro, tanto económico como de tiempo, al permitir evaluar la situación de las aguas «a pie de río».

\section{AGRADECIMIENTOS}

Este trabajo se han beneficiado del Proyecto CAICYT n ${ }^{\circ} 3243183$ y del proyecto LUCDEME (ICONA).

\section{Bibliografia}

Alba-Tercedor, J. \& F. Jiménez Millán, 1985. Biología y ecología de insectos indicadores de la calidad de las aguas corrientes. Estudio de aguas del Río Guadalfeo y su Cuenca. (Calidad de las aguas del Río Guadalfeo a lo largo del $\boldsymbol{a} \tilde{n} \boldsymbol{o})$. Proyecto finalizado, realizado dentro de los convenios entre las universidades andaluzas y el ICONA. Univ. de Granada. 133 pp.
Alba-Tercedor, J. \& F. Jiménez Mullán, 1987. Evaluación de las variaciones estacionales de la calidad de las aguas del Río Guadalfeo, basada en el estudio de las comunidades de macroinvertebrados acuáticos y de los factores físicoquímicos, En: LUCDEME III. ICONA. Monografía 48: 1-91. Madrid.

Alba-Tercedor, J. \& A. Sánchez-Ortega, \& I. GuisasoLA, en prensa. Caracterización de los cursos permanentes de agua de la cuenca del Río Adra: Factores físicoquímicos, macroinvertebrados acuáticos y calidad de las aguas. En: Estudio del medio natural de la cuenca del Río Adra. LUCDEME. ICONA.

CHANDLER, J.R., 1970. A biological approach to water quality management. Water Poll. Control., 69: 415-422.

DiXON, W.J. (ed.) 1981. BMDP statistical software 1981. Univ. California press, Berkeley, 734 págs.

Domezain, A., I. Guisasola \& J. Alba-Tercedor, en prensa. Estudio de la incidencia de una piscifactoría en las comunidades de macroinvertebrados acuáticos. Aplicación de índices bióticos. Limnetica.

Ghet1 , P.F. \& G. BonAZZI, 1980. Biological water assesment methods. Final report. Comission of the European Communities, 2: 1-39, ENV/729/80.

Ghetti, P.F., F. Bernini, G., Bonazzi, A. Cunsolo, \& U. RAVANETT, 1983. Mappagio biologico di qualità dei corsi d'acqua della provincia di Piacenza. Administrazione Prov. di Piacenza, CNR progetto finalizzato «Promozione della qualita dell'ambiente-Progetto di trasferimento», 20 págs.

García de Jalón, D. \& M. González del Tánago, 1986. Métodos Biológicos para el estudio de la calidad de las aguas. Aplicación a la cuenca del Duero. ICONA, Monografía 45. Madrid. 244 págs.

González del Tánago M., D. Garcta de Jalón, \& M., I. ECORO, 1979. Estudio sobre la fauna de macroinvertebrados de los ríos: Cigüela, Záncara y Córcoles; aplicación de índices biológicos para el estudio de la calidad de las aguas. Bol. de la Estación Central de Ecología, ICONA, 8 (15): 45-59.

HELLAWELL, J.M. 1978. Biological surveillance of rivers. Water Research Center, Stevenage, 332 pp.

HulBerT, S.H. 1971. The nonconcept of species diversity: a critique and alternative parameters. Ecology. 52: 557-586. 
LOPez-LlaneZA, J. 1984. Estudio de la calidad del agua en el Río Nalón y su cuenca. (Comparación de índices de calidad en aguas dulces). Consejería de Ordenación del Territorio, Vivienda y Medio Ambiente. Gobierno del Principado de Asturias. $128 \mathrm{pp}$.

MARGALEF, R. 1951. Diversidad de especies en las comunidades naturales. P. Inst. Biol. Apl., 9: 15-27.

Mcintosh, R.P. 1967. An index of diversity and the relation of certain concepts to diversity. Ecology, 48: 392-404.

Prat, N., M.A. Puig, G. González \& X. Millet., 1984. Chironomid longitudinal distribution and rnacroinvertebrates diversity along the Llobregat River (NE Spain). Mem. Amer. Ent. Soc., 34: 267-278.

Prat, N., M.A. Puig, G. González, M.F. Tort \& M EsTRADA, 1984. Llobregat. En: Ecology of European rivers: pp. 527-552. B.A. Whitton (ed.). Blackwell Scientific Publ. Oxford.

Prat, N., G. González, X. Millet \& M.A. Puig, 1985. El Foix entre l'eixutesa i la contaminació. Estudis i monografies, Servei del medi ambient, Diputació de Barcelona, 11, $92 \mathrm{pp}$
Shannon, C.E. \& W. WEAver, 1949. The mathematical theory of communiation. The Univ. of Illinois Press, Urbana, IL.

SiMPSON, E.H. 1949. Measurement of diversity. Nature, 163 (4148): 668.

TuFfery, G. \& J. Verneaux, 1967. Méthode de détermination de la qualité biologique des eaux courantes. Exploitation codifié des inventaires de la faune du fond. Trav. Sect. Tech. P. et. P., C.E.R.A.F.E.R., París, 23 pp.

WARD, J.V. \& J.A. STANDFORD, 1983. The intermediate disturbance hypothesis: and explanation for biotic diversity patterns in lotic ecosysterns. En: The dynamics of lotic ecosystems. Fontaine, T.D. y S.M. (eds.). Ann Arbor Michigan.

WashingtON, H.G. 1984. Diversity and similarity indices. A review with special relevance to aquatic ecosystems. Water Res., 18 (6): 653-694.

WILHM, J.L. \& T.C. DORRIS, 1968. Biological parameters of water quality. Bioscience, 18: 477-481.

WooDiwiss, F.S. 1978. Biological Water Assesment Methods. Severn-Trent River Authorities, U.K. 